\title{
ABOUT DYNAMIC STABILITY OF DEFORMABLE ELEMENTS OF VIBRATION SYSTEMS
}

\author{
Petr A. Velmisov \\ Department of higher mathematics \\ Ulyanovsk State Technical University \\ Russia \\ velmisov@ulstu.ru
}

\author{
Andrey V. Ankilov \\ Department of higher mathematics \\ Ulyanovsk State Technical University \\ Russia \\ ankil@ulstu.ru
}

Article history:

Received 29.10.2019, Accepted 28.11.2019

\begin{abstract}
The dynamics and stability of the elastic elements of vibration devices, modeled by a channel containing elastic elements, are investigated. Inside the channel flows a stream of stirred liquid. The model of the device with two elastic elements is considered. The solution of the aerohydrodynamic part of the problem, based on the methods of the theory of functions of a complex variable, is given. The solution of the original problem is reduced to the study of a coupled system of partial differential equations for the deformations of elements, which makes it possible to study their dynamics. On the basis of the constructed functional for this system, the sufficient conditions of stability are obtained. The conditions impose restrictions on the parameters of the mechanical system. Based on the Galerkin method, the numerical experiments for specific examples of mechanical systems were carried out, confirming the reliability of the investigations. A special case of the model of device with one elastic element is considered. Based on this case, a comparison with the model of the vibration device considered earlier is made.
\end{abstract}

\section{Key words}

Fluid-structure interaction; Elastic plate; Stability; Dynamics; Functional.

\section{Introduction}

For the design and exploitation of structures, devices, mechanisms for various applications, interacting with liquid, an important problem is to ensure the reliability of their functioning and durability. Similar problems are common to many branches of engineering. In particular, such problems arise in missilery, aircraft construction, instrumentation, and so on. A stability investigation of the deformable elements has essential value in the calculation of structures that interact with the liquid, as the impact of the liquid may lead to its loss. Examples of the loss of dynamic stability include: the flutter of an airplane wing; panel flutter of plates and shells that are streamlined by a gas or liquid, for example, flutter of an aircraft or rocket skin panel; shear flutter of turbine blades and propellers; oscillations of wires, ducts, suspension bridges, and so on.

At the same time, for the operation of some technical devices, the phenomenon of excitation of vibrations during aero-hydrodynamic effects, mentioned above as negative, is necessary. Examples of such devices related to the vibration technique and used to intensify technological processes are devices for preparing homogeneous mixtures and emulsions, in particular, devices for supplying coolant to the treatment area (see, for example, [Velmisov et al., 1996]). The main part of a wide class of such devices is a flow channel, on the walls of which (or inside it) the elastic elements are located. The operation of such devices is based on the vibration of elastic elements during the flow of liquid inside the channels.

Thus, for designing of the structures and devices interacting with the liquid, it is necessary to solve problems related to the investigation of stability required for their functioning and operational reliability.

Many experimental and theoretical studies have been devoted to stability analysis of elastic bodies interacting with gas and liquid. Among them we should note [Abdelbaki et al., 2019; Blinkov et al., 2018; Kheiri and Paidoussis, 2015; Kontzialis et al., 2017; Moditis et al., 2016; Mogilevich et al., 2017; Mogilevich et al., 2018; Moshkelgosha et al., 2017; Zvyagin and Gur'ev, 2017] and many others. Among the works of the authors of this article about fluid-structure interaction, note the monograph and articles [Ankilov and Velmisov, 2013; 
Ankilov and Velmisov, 2016; Velmisov and Ankilov, 2017; Velmisov and Ankilov, 2018; Velmisov and Ankilov, 2018].

The goal of this work is to analyze stability of elastic body correspond to the Lyapunov concept of stability of dynamical systems. The problem can be formulated as follows: for any values of the parameters characterizing the system "liquid-solid" (the main parameters are the flow velocity, strength and inertial characteristics of the body, compressive or tensile forces, friction forces) determine whether small deformation of bodies at the initial time $t=0$ (i.e. a small initial deviations from the equilibrium position) correspond to small deformations at any time $t>0$.

In the work, the problems of the dynamics and stability of the elastic elements of the vibration device are investigated. The study of the dynamic stability of the working elements of vibrating device is necessary for optimal control of the parameters of the mechanical system in order to increase the efficiency of its functioning. The device is a flow channel with deformable elements, simulated by elastic plates, which can be located both on the walls of the channel and inside it. The number and location of elements are arbitrary. A subsonic flow of an ideal compressible or incompressible medium flows through the channel. To study the dynamics of elastic elements, nonlinear equations are used that describe the longitudinal-transverse oscillations of elastic plates. The aerohydrodynamic load is determined from the asymptotic equations of aero-hydromechanics. At the inlet and outlet of the channel, either the laws of pressure change, or the velocity potential of the liquid, or the longitudinal components of the velocity of the liquid are given. At the inlet to the channel, the flow velocity of the liquid is assumed to be constant and directed along the channel axis (Figure 1).

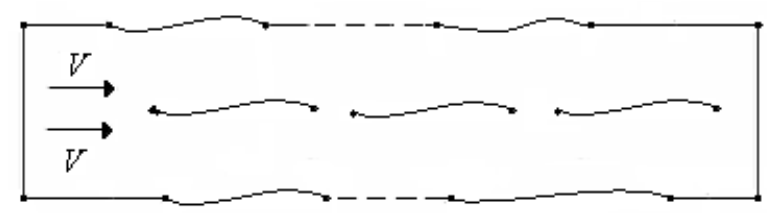

Figure 1. Model of the vibration device.

As an example, let us consider a mathematical model of a hydrodynamic emitter - a vibration device designed to prepare homogeneous mixtures and emulsions. The main components of the device are two elastic elements located on the walls of the flow channel. The oscillations of elastic elements lead to mixing of the inhomogeneous medium supplied to this channel.

\section{Model of Device with Two Elastic Elements}

\subsection{Mathematical Model}

A flat flow in a straight line channel $J=\left\{(x, y) \in R^{2}\right.$ : $\left.0<y<y_{0}\right\}$ is considered (Figure 2).

It is assumed that the longitudinal size of the channel considerably exceeds its transverse size, which leads to the absence of disturbances at a point sufficiently far from the elements. The velocity of the undisturbed flow will be considered equal $V$ and directed along the axis $O x$. Suppose that the elastic parts are located on the walls $y=0$ and $y=y_{0}$ at $x \in[a, b]$ (Figure 2).

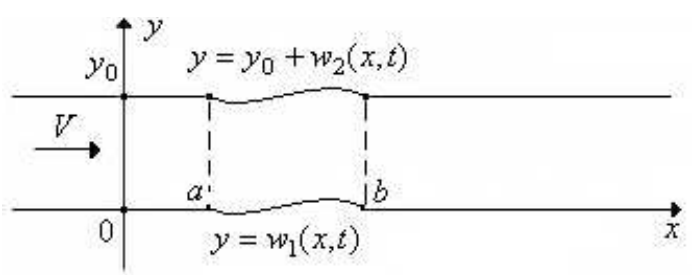

Figure 2. A channel whose walls contain deformable elements

We introduce the following notation: $u_{i}(x, t), w_{i}(x, t)$ are elastic displacement of insert plates in the direction of axes $O x$ and $O y$ walls $y=0$ at $i=1$ and $y=y_{0}$ at $i=2 ; \phi(x, y, t)$ is potential of the velocity of the disturbed flow.

Functions $w_{i}(x, t) \in C^{4,2}\left\{[a, b] \times R^{+}\right\}$, i.e. it belongs to four times continuously differentiable functions with respect to the variable $x$ on the interval $(a, b)$ and twice continuously differentiable with respect to the variable $t$ at $t \geq 0$ and takes real values.

Functions $u_{i}(x, t) \in C^{2,2}\left\{[a, b] \times R^{+}\right\}$, i.e. it belongs to twice continuously differentiable functions with respect to the variable $x$ on the interval $(a, b)$ and twice continuously differentiable with respect to the variable $t$ at $t \geq 0$ and takes real values.

Function $\phi(x, y, t) \in C^{2,1}\left\{J \times R^{+}\right\}$, i.e. it belongs to twice continuously differentiable functions with respect to the variables $x, y$ in the area $J$ and continuously differentiable with respect to the variable $t$ at $t \geq 0$ and takes real values.

In a model of an ideal incompressible medium the potential $\phi$ of the disturbed flow satisfies the Laplace equation:

$$
\Delta \phi \equiv \phi_{x x}+\phi_{y y}=0, \quad(x, y) \in J .
$$

The linearized boundary conditions arising from the condition of impermeability have form:

$$
\begin{gathered}
\phi_{y}\left(x, y_{i}, t\right)=0, x \in(-\infty, a] \bigcup[b,+\infty) ; \\
\phi_{y}\left(x, y_{i}, t\right)=\dot{w}_{i}(x, t)+V w_{i}^{\prime}(x, t), x \in(a, b),
\end{gathered}
$$


where $i=1,2, y_{1}=0, y_{2}=y_{0}$. The conditions for the absence of disturbances at an infinitely distant point:

$$
\left(\phi_{x}^{2}+\phi_{y}^{2}+\phi_{t}^{2}\right)_{x= \pm \infty}=0, y \in\left(0, y_{0}\right) .
$$

The boundary conditions corresponding to rigid fixing of the ends of the plates:

$$
\begin{array}{r}
u_{i}(a, t)=w_{i}(a, t)=w_{i}^{\prime}(a, t)=u_{i}(b, t)= \\
=w_{i}(b, t)=w_{i}^{\prime}(b, t)=0, i=1,2 .
\end{array}
$$

Generalizing the nonlinear equations of small oscillations of elastic plates obtained in [Shmidt, 1978], we write them in the form

$$
\left\{\begin{array}{l}
-E_{i} F_{i}\left(u_{i}^{\prime}(x, t)+\frac{1}{2}{w_{i}^{\prime}}^{2}(x, t)\right)^{\prime}+ \\
+M_{i} \ddot{u}_{i}(x, t)=0, \\
-E_{i} F_{i}\left[w_{i}^{\prime}(x, t)\left(u_{i}^{\prime}(x, t)+\frac{1}{2} w_{i}^{\prime 2}(x, t)\right)\right]^{\prime}+ \\
+M_{i} \ddot{w}_{i}(x, t)+D_{i} w_{i}^{\prime \prime \prime \prime}(x, t)+ \\
+N_{i}(t) w_{i}^{\prime \prime}(x, t)+\beta_{0 i} w_{i}(x, t)+\beta_{1 i} \dot{w}_{i}(x, t)+ \\
+\beta_{2 i} I_{i} \dot{w}_{i}^{\prime \prime \prime \prime}(x, t)=(-1)^{i}\left(P_{0}-P_{*}-\right. \\
\left.-\rho\left(\phi_{t}\left(x, y_{i}, t\right)+V \phi_{x}\left(x, y_{i}, t\right)\right)\right), \\
i=1,2, x \in(a, b) .
\end{array}\right.
$$

The indices $x, y, t$ below denote partial derivatives with respect to $x, y, t$; the bar and the point denote the partial derivatives with respect to $x$ and $t$, respectively; $\rho$ is density of liquid; $I_{i}=h_{i}^{3} /\left(12\left(1-\nu_{i}^{2}\right)\right)$; $D_{i}=E_{i} I_{i}$ are flexural stiffness of plates; $h_{i}$ are thicknesses of plates; $M_{i}=h_{i} \rho_{p l}$ are linear masses of plates; $F_{i}=h_{i} /\left(1-\nu_{i}^{2}\right) ; E_{i}, \rho_{p l}$ are elasticity modulus and the linear density of the plates; $\nu_{i}$ are Poisson coefficients; $N_{i}(t)$ are compressing $\left(N_{i}>0\right)$ or tensile $\left(N_{i}<0\right)$ forces of the plates; $\beta_{2 i}, \beta_{1 i}$ are coefficients of internal and external damping; $\beta_{0 i}$ are stiffness coefficients of the bases (beds); $P_{0}$ is pressure in a uniform flow; $P_{*}$ is external distributed load acting on the channel walls.

Compressive (tensile) forces $N_{i}(t)$ elements may depend on time. For example, if a non-stationary heat exposure to the plate the $N_{i}(t)$ is as follows:

$$
N_{i}(t)=N_{0 i}+\frac{E_{i} \alpha_{T i}}{1-\nu_{i}} \int_{-h_{i} / 2}^{h_{i} / 2} T_{i}(z, t) d z
$$

where $\alpha_{T i}$ are the temperature coefficients of linear expansion, $T_{i}(z, t)$ are the laws of temperature change over the thickness of the plates, $N_{0 i}$ are the constant components of forces generated when fixing plates.

A nonlinear boundary value problem (1) - (6) was obtained for determining five unknown functions - the deformations of elastic elements $u_{i}(x, t), w_{i}(x, t), i=1,2$ and the velocity potential $\phi(x, y, t)$ of the liquid.

\subsection{Determination of the Flow Force}

In the region $J$, we introduce the complex potential $W=f(z, t)=\phi+i \psi, z=x+i y$ and consider the analytic function $f_{z}(z, t)=\phi_{x}+i \psi_{x}=\phi_{x}-i \phi_{y}$. Using the function $\zeta=-e^{-\pi z / y_{0}}$, we conformally map the strip $J$ to the upper half-plane $H=\{\zeta: \operatorname{Im} \zeta>0\}$ of the complex variable $\zeta=\xi+i \eta$. In this case, the segments $[-\alpha,-\beta],[\beta, \alpha]$ on the real axis will correspond to the elastic plates, where $\beta=e^{-\pi b / y_{0}}, \alpha=e^{-\pi a / y_{0}}$. According to the boundary conditions (2), (3) we will have

$$
\begin{gathered}
\operatorname{Re}\left\{i f_{z}(z(\zeta), t)\right\}=\phi_{y}= \\
=\left\{\begin{array}{l}
0, \xi \notin[-\alpha,-\beta] \cup[\beta, \alpha], \\
\bar{w}_{1}(x(\xi), t), \xi \in[-\alpha,-\beta], \\
\bar{w}_{2}(x(\xi), t), \xi \in[\beta, \alpha],
\end{array}\right.
\end{gathered}
$$

where

$\bar{w}_{1}=\dot{w}_{1}+V w_{1}^{\prime}, \bar{w}_{2}=\dot{w}_{2}+V w_{2}^{\prime}, x(\xi)=-\frac{y_{0}}{\pi} \ln |\xi|$.

According to (4), applying the Schwartz integral, we obtain

$$
\begin{aligned}
f_{z}(z(\zeta), t) & =-\frac{1}{\pi}\left(\int_{-\alpha}^{-\beta} \frac{\bar{w}_{1}(x(\tau), t)}{\tau-\zeta} d \tau+\right. \\
& \left.+\int_{\beta}^{\alpha} \frac{\bar{w}_{2}(x(\tau), t)}{\tau-\zeta} d \tau\right)+C(t),
\end{aligned}
$$

where $C(t)$ is arbitrary function of the variable $t$.

As $f_{z}=\phi_{x}-i \phi_{y} \rightarrow C(t)$ at $\zeta \rightarrow \infty(x \rightarrow-\infty)$, then from (4) it follows that $C(t) \equiv 0$. By virtue of the same condition at $\zeta \rightarrow 0(x \rightarrow+\infty)$, from (8) we have

$$
\int_{-\alpha}^{-\beta} \frac{\bar{w}_{1}(x(\tau), t)}{\tau} d \tau+\int_{\beta}^{\alpha} \frac{\bar{w}_{2}(x(\tau), t)}{\tau} d \tau=0 .
$$

or, given that $x(\tau)=-\frac{y_{0}}{\pi} \ln |\tau|$,

$$
\int_{a}^{b}\left(\frac{\partial w_{1}}{\partial t}+V \frac{\partial w_{1}}{\partial x}\right) d x=\int_{a}^{b}\left(\frac{\partial w_{2}}{\partial t}+V \frac{\partial w_{2}}{\partial x}\right) d x
$$

According to the boundary conditions (5) we obtain

$$
\int_{a}^{b} \frac{\partial w_{1}}{\partial t} d x=\int_{a}^{b} \frac{\partial w_{2}}{\partial t} d x
$$

The physical meaning (9) is that the gas flow through the boundary of region $J$ equal to zero, which corresponds to the model of an incompressible medium. 
Further, since $W_{\zeta}=f_{z} \frac{d z}{d \zeta}=-f_{z} \frac{y_{0}}{\pi \zeta}$, integrating by $\zeta$ and differentiating by $t$, according to (4) we obtain:

$$
\begin{array}{r}
W_{t}=f_{t}(z(\zeta), t)=-\frac{y_{0}}{\pi^{2}}\left(\int_{-\alpha}^{-\beta} \frac{\bar{w}_{1 t}(x(\tau), t)}{\tau} \times \quad(10)\right. \\
\left.\times \ln (\tau-\zeta) d \tau+\int_{\beta}^{\alpha} \frac{\bar{w}_{2 t}(x(\tau), t)}{\tau} \ln (\tau-\zeta) d \tau\right)+ \\
+C_{1}(t),
\end{array}
$$

where $C_{1}(t)$ is arbitrary function of the variable $t$. According to (4) the condition $\phi_{t} \rightarrow 0$ at $\zeta \rightarrow \infty(x \rightarrow$ $-\infty)$ is also satisfied, therefore from (10) it follows that $\operatorname{Re}_{1}(t) \equiv 0$. And from condition $\phi_{t} \rightarrow 0$ at $\zeta \rightarrow 0(x \rightarrow+\infty)$ we have

$$
\begin{gathered}
\int_{-\alpha}^{-\beta} \frac{\bar{w}_{1 t}(x(\tau), t)}{\tau} \ln (-\tau) d \tau+ \\
+\int_{\beta}^{\alpha} \frac{\bar{w}_{2 t}(x(\tau), t)}{\tau} \ln (\tau) d \tau=0,
\end{gathered}
$$

or, given that $x(\tau)=-\frac{y_{0}}{\pi} \ln |\tau|$,

$$
\int_{a}^{b} x\left(\frac{\partial w_{1}}{\partial t}+V \frac{\partial w_{1}}{\partial x}\right) d x=\int_{a}^{b} x\left(\frac{\partial w_{2}}{\partial t}+V \frac{\partial w_{2}}{\partial x}\right) d x .
$$

Using the integral representations (8), (10), we transform the right-hand side of the second equation of system (6). To this end, in (8), (10) we pass to the limits at $\zeta \rightarrow \xi \in(-\alpha,-\beta) \quad(z \rightarrow x+i \cdot 0, \quad a<x<b)$ and $\zeta \rightarrow \xi \in(\beta, \alpha)\left(z \rightarrow x+i \cdot y_{0}, a<x<b\right)$. Applying the Sokhotsky's formula, we obtain

$$
\begin{gathered}
\rho\left(\phi_{t}\left(x, y_{i}, t\right)+V \phi_{x}\left(x, y_{i}, t\right)\right)= \\
=-\frac{y_{0} \rho}{\pi^{2}}\left(\int_{-\alpha}^{-\beta} \frac{\partial \bar{w}_{1}(x(\tau), t)}{\partial t} \frac{\ln |\tau-\xi|}{\tau} d \tau+\right. \\
\left.+\int_{\beta}^{\alpha} \frac{\partial \bar{w}_{2}(x(\tau), t)}{\partial t} \frac{\ln |\tau-\xi|}{\tau} d \tau\right)- \\
-\frac{\rho V}{\pi}\left(\int_{-\alpha}^{-\beta} \frac{\bar{w}_{1}(x(\tau), t)}{\tau-\xi} d \tau+\int_{\beta}^{\alpha} \frac{\bar{w}_{2}(x(\tau), t)}{\tau-\xi} d \tau\right) \\
\xi=(-1)^{i} e^{-\pi x / y_{0}}, i=1,2, a<x<b
\end{gathered}
$$

Substituting $\xi, \tau$, we write the expressions (11) in the form

$$
\begin{array}{r}
\rho(-1)^{i}\left(\phi_{t}\left(x, y_{i}, t\right)+V \phi_{x}\left(x, y_{i}, t\right)\right)= \\
=\frac{\rho V}{\pi}\left(\int_{a}^{b}\left(\dot{w}_{i}(\tau, t)+V w_{i}^{\prime}(\tau, t)\right) \frac{\partial K_{1}(\tau, x)}{\partial x} d \tau-\right. \\
\left.-\int_{a}^{b}\left(\dot{w}_{3-i}(\tau, t)+V w_{3-i}^{\prime}(\tau, t)\right) \frac{\partial K_{2}(\tau, x)}{\partial x} d \tau\right)+ \\
+\frac{\rho}{\pi}\left(\int_{a}^{b}\left(\ddot{w}_{i}(\tau, t)+V \dot{w}_{i}^{\prime}(\tau, t)\right) K_{1}(\tau, x) d \tau-\right. \\
\left.-\int_{a}^{b}\left(\ddot{w}_{3-i}(\tau, t)+V \dot{w}_{3-i}^{\prime}(\tau, t)\right) K_{2}(\tau, x) d \tau\right), \\
i=1,2, \quad x \in(a, b),
\end{array}
$$

where

$$
\begin{gathered}
K_{1}(\tau, x)=\ln \left|\frac{2 \exp \left(\frac{-\pi a}{y_{0}}\right)}{\exp \left(\frac{-\pi \tau}{y_{0}}\right)-\exp \left(\frac{-\pi x}{y_{0}}\right)}\right|, \\
K_{2}(\tau, x)=\ln \left|\frac{2 \exp \left(\frac{-\pi a}{y_{0}}\right)}{\exp \left(\frac{-\pi \tau}{y_{0}}\right)+\exp \left(\frac{-\pi x}{y_{0}}\right)}\right|, \\
K(\tau, x)=K_{1}(\tau, x)-K_{2}(\tau, x), \quad \tau \neq x .
\end{gathered}
$$

It is easy to see that the kernels are symmetric $K_{i}(\tau, x)=K_{i}(x, \tau), i=1,2, K(\tau, x)=K(x, \tau)$. Besides $K_{1}(\tau, x) \geq K(\tau, x) \geq K_{2}(\tau, x) \geq 0$. The expressions (12) are obtained for any methods of fixing elastic plates.

Substituting (12) into (6), we obtain a system of differential equations with four unknown functions $u_{1}(x, t)$, $w_{1}(x, t), u_{2}(x, t), w_{2}(x, t)$ :

$$
\begin{array}{r}
-E_{i} F_{i}\left(u_{i}^{\prime}(x, t)+\frac{1}{2}{w_{i}^{\prime}}^{2}(x, t)\right)^{\prime}+M_{i} \ddot{u}_{i}(x, t)=0, \\
-E_{i} F_{i}\left[w_{i}^{\prime}(x, t)\left(u_{i}^{\prime}(x, t)+\frac{1}{2}{w_{i}^{\prime}}^{2}(x, t)\right)\right]^{\prime}+ \\
+M_{i} \ddot{w}_{i}(x, t)+D_{i} w_{i}^{\prime \prime \prime \prime}(x, t)+N_{i}(t) w_{i}^{\prime \prime}(x, t)+ \\
+\beta_{0 i} w_{i}(x, t)+\beta_{1 i} \dot{w}_{i}(x, t)+\beta_{2 i} I_{i} \dot{w}_{i}^{\prime \prime \prime \prime}(x, t)=(13 \\
=-\frac{\rho V}{\pi}\left(\int_{a}^{b}\left(\dot{w}_{i}(\tau, t)+V w_{i}^{\prime}(\tau, t)\right) \frac{\partial K_{1}(\tau, x)}{\partial x} d \tau-\right. \\
\left.-\int_{a}^{b}\left(\dot{w}_{3-i}(\tau, t)+V w_{3-i}^{\prime}(\tau, t)\right) \frac{\partial K_{2}(\tau, x)}{\partial x} d \tau\right)- \\
-\frac{\rho}{\pi}\left(\int_{a}^{b}\left(\ddot{w}_{i}(\tau, t)+V \dot{w}_{i}^{\prime}(\tau, t)\right) K_{1}(\tau, x) d \tau-\right.
\end{array}
$$




$$
\begin{array}{r}
\left.-\int_{a}^{b}\left(\ddot{w}_{3-i}(\tau, t)+V \dot{w}_{3-i}^{\prime}(\tau, t)\right) K_{2}(\tau, x) d \tau\right), \\
i=1,2, x \in(a, b) .
\end{array}
$$

The system (13) is homogeneous and obtained under the assumption that $P_{0}=P_{*}$.

\subsection{Stability Investigation}

Introduce the following notation: $\lambda_{1 i}, \eta_{1 i}$ are the smallest eigenvalues of the boundary value problems for the equations $\psi^{\prime \prime \prime \prime}=-\lambda \psi^{\prime \prime}, \psi^{\prime \prime \prime \prime}=\eta \psi, x \in[a, b]$ with boundary conditions corresponding (5) for the functions $w_{i}(x, t), i=1,2$;

$$
\begin{aligned}
& G=\sup _{x \in[a, b]} \int_{a}^{b}\left|K_{1}(\tau, x)+g_{1}(x)+g_{1}(\tau)\right| d \tau, \\
& K=\sup _{x \in[a, b]} \int_{a}^{b}\left|K_{2}(\tau, x)+g_{2}(x)+g_{2}(\tau)\right| d \tau,
\end{aligned}
$$

where $g_{1}(x), g_{2}(x)$ are the arbitrary functions integrable over a segment $[a, b]$ chosen for reasons of attaining the smallest possible values $G, K$. Further an example of the choice of functions $g_{1}(x), g_{2}(x)$ is given.

Theorem 2.1. Let the conditions

$$
\begin{gathered}
\beta_{0 i} \geq 0, \quad \beta_{1 i}+\beta_{2 i} I_{i} \eta_{1 i} \geq 0, \quad \dot{N}_{i}(t) \geq 0, \\
M_{i} \geq \frac{2 \rho K}{\pi}, N_{i}(t)<D_{i} \lambda_{1 i}-\frac{\rho V^{2}(G+K)}{\pi}
\end{gathered}
$$

be fulfilled. Then the solutions $w_{i}(x, t)(i=1,2)$ of the system of equations (13) are stable with respect to the perturbations of the initial values $w_{i}(x, 0), \dot{u}_{i}(x, 0)$, $\dot{w}_{i}(x, 0), u_{i}^{\prime}(x, 0), w_{i}^{\prime}(x, 0), w_{i}^{\prime \prime}(x, 0)(i=1,2)$, if the functions $u_{i}(x, t), w_{i}(x, t)(i=1,2)$ satisfy the boundary conditions (5).

Proof. We introduce the functional

$$
\begin{gathered}
\Phi(t)=\sum_{i=1}^{2} \int_{a}^{b}\left\{E_{i} F_{i}\left(u_{i}^{\prime}(x, t)+\frac{1}{2}{w_{i}^{\prime}}^{2}(x, t)\right)^{2}+\right. \\
+M_{i}\left(\dot{u}_{i}^{2}(x, t)+\dot{w}_{i}^{2}(x, t)\right)+D_{i}{w_{i}^{\prime \prime}}^{2}(x, t)- \\
\left.-N_{i}(t){w_{i}^{\prime}}^{2}(x, t)+\beta_{0 i} w_{i}^{2}(x, t)\right\} d x+\sum_{i=1}^{3}\left(I_{i}(t)+J_{i}(t)\right),
\end{gathered}
$$

where

$$
J_{i}(t)=-\frac{\rho V^{2}}{\pi} \int_{a}^{b} d x \int_{a}^{b} w_{i}^{\prime}(x, t) w_{i}^{\prime}(\tau, t) K_{1}(\tau, x) d \tau,
$$

$$
\begin{gathered}
I_{i}(t)=\frac{\rho}{\pi} \int_{a}^{b} d x \int_{a}^{b} \dot{w}_{i}(x, t) \dot{w}_{i}(\tau, t) K_{1}(\tau, x) d \tau, i=1,2, \\
J_{3}(t)=\frac{2 \rho V^{2}}{\pi} \int_{a}^{b} d x \int_{a}^{b} w_{1}^{\prime}(x, t) w_{2}^{\prime}(\tau, t) K_{2}(\tau, x) d \tau, \\
I_{3}(t)=-\frac{2 \rho}{\pi} \int_{a}^{b} d x \int_{a}^{b} \dot{w}_{1}(x, t) \dot{w}_{2}(\tau, t) K_{2}(\tau, x) d \tau .
\end{gathered}
$$

Find the derivative of $\Phi$ by $t$

$$
\begin{gathered}
\dot{\Phi}(t)=2 \sum_{i=1}^{2} \int_{a}^{b}\left\{E_{i} F_{i}\left(\left(u_{i}^{\prime}+\frac{1}{2} w_{i}^{\prime 2}\right)^{2}\right)_{t}+\right. \\
+M_{i} \dot{u}_{i} \ddot{u}_{i}+M_{i} \dot{w}_{i} \ddot{w}_{i}+D_{i} w_{i}^{\prime \prime} \dot{w}_{i}^{\prime \prime}-\frac{1}{2} \dot{N}_{i}(t) w_{i}^{\prime 2}- \\
\left.-N_{i}(t) w_{i}^{\prime} \dot{w}_{i}^{\prime}+\beta_{0 i} w_{i} \dot{w}_{i}\right\} d x+\sum_{i=1}^{3}\left(\dot{I}_{i}+\dot{J}_{i}\right),
\end{gathered}
$$

We will integrate by parts taking into account the boundary conditions (6):

$$
\begin{gathered}
\int_{a}^{b} \dot{w}_{i}\left[w_{i}^{\prime}\left(u_{i}^{\prime}+\frac{1}{2} w_{i}^{\prime 2}\right)\right]^{\prime} d x+\int_{a}^{b} \dot{u}_{i}\left(u_{i}^{\prime}+\frac{1}{2} w_{i}^{\prime 2}\right)^{\prime} d x= \\
=\frac{1}{2}\left(\int_{a}^{b}\left(u_{i}^{\prime}+\frac{1}{2} w_{i}^{\prime 2}\right)^{2} d x\right)_{t}, \int_{a}^{b} \dot{w}_{i} w_{i}^{\prime \prime \prime \prime} d x= \\
=\int_{a}^{b} \dot{w}_{i}^{\prime \prime} w_{i}^{\prime \prime} d x, \quad \int_{a}^{b} \dot{w}_{i} \dot{w}_{i}^{\prime \prime \prime \prime} d x=\int_{a}^{b} \dot{w}_{i}^{\prime \prime 2} d x, \\
\int_{a}^{b} \dot{w}_{i} w_{i}^{\prime \prime} d x=-\int_{a}^{b} \dot{w}_{i}^{\prime} w_{i}^{\prime} d x, \\
\int_{a}^{b} d x \int_{a}^{b} \dot{w}_{i}(x, t) \dot{w}_{i}(\tau, t) \frac{\partial K_{1}(\tau, x)}{\partial x} d \tau= \\
\int_{a}^{b} d x \int_{a}^{b} \dot{w}_{i}(x, t) \dot{w}_{i}^{\prime}(\tau, t) K_{1}(\tau, x) d \tau, i=1,2 .
\end{gathered}
$$

Similar to the last equality, it is possible to obtain equalities for all integrals with integrands containing $\frac{\partial K_{i}(\tau, x)}{\partial x}, i=1,2$. 
Taking into account the symmetry of the kernels $K_{1}(\tau, x)=K_{1}(x, \tau), K_{2}(\tau, x)=K_{2}(x, \tau)$, we obtain

$$
\begin{array}{r}
\dot{J}_{i}(t)=-\frac{2 \rho V^{2}}{\pi} \int_{a}^{b} d x \int_{a}^{b} \dot{w}_{i}^{\prime}(x, t) w_{i}^{\prime}(\tau, t) K_{1}(\tau, x) d \tau, \\
\dot{I}_{i}(t)=\frac{2 \rho}{\pi} \int_{a}^{b} d x \int_{a}^{b} \dot{w}_{i}(x, t) \ddot{w}_{i}(\tau, t) K_{1}(\tau, x) d \tau, \\
\dot{I}_{3}(t)=-\frac{2 \rho}{\pi} \int_{a}^{b} d x \int_{a}^{b} \dot{w}_{1}(x, t) \ddot{w}_{2}(\tau, t) K_{2}(\tau, x) d \tau- \\
\quad-\frac{2 \rho}{\pi} \int_{a}^{b} d x \int_{a}^{b} \dot{w}_{2}(x, t) \ddot{w}_{1}(\tau, t) K_{2}(\tau, x) d \tau, \\
\dot{J}_{3}(t)=\frac{2 \rho V^{2}}{\pi} \int_{a}^{b} d x \int_{a}^{b} \dot{w}_{1}^{\prime}(x, t) w_{2}^{\prime}(\tau, t) K_{2}(\tau, x) d \tau+ \\
+\frac{2 \rho V^{2}}{\pi} \int_{a}^{b} d x \int_{a}^{b} \dot{w}_{2}^{\prime}(x, t) w_{1}^{\prime}(\tau, t) K_{2}(\tau, x) d \tau .
\end{array}
$$

According to the obtained equalities for functions $u_{i}(x, t), w_{i}(x, t), i=1,2$, that are solutions of the system of equations (13), the equality (19) takes the form:

$$
\begin{aligned}
& \dot{\Phi}(t)= \\
& =-2 \sum_{i=1}^{2} \int_{a}^{b}\left\{\beta_{1 i} \dot{w}_{i}^{2}+\beta_{2 i} I_{i} \dot{w}_{i}^{\prime \prime 2}+\frac{1}{2} \dot{N}_{i}(t) w_{i}^{\prime 2}\right\} d x .
\end{aligned}
$$

Consider boundary value problems for equations $\psi^{\prime \prime \prime \prime}=-\lambda \psi^{\prime \prime}, \psi^{\prime \prime \prime \prime}=\eta \psi, x \in(a, b)$ with boundary conditions (5) for the functions $w_{i}(x, t)$. These problems are positively defined and fully defined. For the function $w_{i}(x, t)$, using the Rayleigh [Kollatz, 1968] and Cauchy-Bunyakovsky inequalities, we obtain estimates

$$
\begin{aligned}
& \int_{a}^{b} \dot{w}_{i}^{\prime \prime 2}(x, t) d x \geq \eta_{1 i} \int_{a}^{b} \dot{w}_{i}^{2}(x, t) d x, \int_{a}^{b} w_{i}^{\prime \prime 2}(x, t) d x \geq \\
& \geq \lambda_{1 i} \int_{a}^{b}{w_{i}^{\prime}}^{2}(x, t) d x, \frac{w_{i}^{2}(x, t)}{(b-a)} \leq \int_{a}^{b}{w_{i}^{\prime}}^{2}(x, t) d x,
\end{aligned}
$$

where $\lambda_{1 i}=\left(\frac{2 \pi}{b-a}\right)^{2}, \eta_{1 i} \approx\left(\frac{4,73}{b-a}\right)^{4}$ are smallest eigenvalues of boundary value problems [Ankilov and Velmisov, 2013].

Using the first inequality in (20), we obtain

$$
\begin{aligned}
& \dot{\Phi}(t) \leq \\
& \leq-2 \sum_{i=1}^{2} \int_{a}^{b}\left\{\left(\beta_{1 i}+\beta_{2 i} I_{i} \eta_{1 i}\right) \dot{w}_{i}^{2}+\frac{1}{2} \dot{N}_{i}(t) w_{i}^{\prime 2}\right\} d x .
\end{aligned}
$$

Let conditions (15) be fulfilled, then we finally obtain $\dot{\Phi}(t) \leq 0$. Integrating from 0 to $t$, we obtain the inequality

$$
\Phi(t) \leq \Phi(0) .
$$

Let us estimate the repeated integrals (18) in the expression for $\Phi(0)$, using the equality $K_{1}(\tau, x)=K(\tau, x)+$ $+K_{2}(\tau, x)$, the inequality proved earlier [Ankilov and Velmisov, 2013]

$$
\int_{a}^{b} d x \int_{a}^{b} f(x) f(\tau) K(\tau, x) d \tau \geq 0,
$$

valid for any continuous functions $f(x)$ on an interval $[a, b]$, an obvious inequality $\pm 2 c d \leq c^{2}+d^{2}$, as well as symmetry and non-negativity of kernels $K_{1}(\tau, x), K_{2}(\tau, x)$, as follows:

$$
\begin{aligned}
& I_{i}(0) \leq \frac{\rho}{\pi} \int_{a}^{b} d x \int_{a}^{b} \dot{w}_{i}^{2}(x, 0) K_{1}(\tau, x) d \tau, i=1,2, \\
& I_{3}(0) \leq \\
& \leq \frac{\rho}{\pi} \int_{a}^{b} d x \int_{a}^{b}\left(\dot{w}_{1}^{2}(x, 0)+\dot{w}_{2}^{2}(x, 0)\right) K_{2}(\tau, x) d \tau \\
& J_{i}(0) \leq \\
& \leq \frac{\rho V^{2}}{\pi} \int_{a}^{b} d x \int_{a}^{b} w_{i}^{\prime 2}(x, 0) K_{2}(\tau, x) d \tau, i=1,2, \\
& J_{3}(0) \leq \frac{\rho V^{2}}{\pi} \times \\
& \times \int_{a}^{b} d x \int_{a}^{b}\left(w_{1}^{\prime 2}(x, 0)+w_{2}^{\prime 2}(x, 0)\right) K_{2}(\tau, x) d \tau .
\end{aligned}
$$

From (17), (18), (23) - (26) follows

$$
\begin{gathered}
\Phi(0) \leq \sum_{i=1}^{2} \int_{a}^{b}\left\{E_{i} F_{i}\left(u_{0 i}^{\prime}+\frac{1}{2} w_{0 i}^{\prime 2}\right)^{2}+M_{i} \dot{u}_{0 i}^{2}+\right. \\
+\left(M_{i}+\frac{\rho\left(K_{0}+G_{0}\right)}{\pi}\right) \dot{w}_{0 i}^{2}+D_{i} w_{0 i}^{\prime \prime}+ \\
\left.+\left(\frac{2 \rho V^{2} G_{0}}{\pi}-N_{i}(0)\right) w_{0 i}^{\prime 2}+\beta_{0 i} w_{0 i}^{2}\right\} d x,
\end{gathered}
$$

where $w_{0 i}=w_{i}(x, 0), \dot{u}_{0 i}=\dot{u}_{i}(x, 0), \dot{w}_{0 i}=\dot{w}_{i}(x, 0)$, $u_{0 i}^{\prime}=u_{i}^{\prime}(x, 0), w_{0 i}^{\prime}=w_{i}^{\prime}(x, 0), w_{0 i}^{\prime \prime}=w_{i}^{\prime \prime}(x, 0)$,

$$
K_{0}=\sup _{x \in(a, b)} \int_{a}^{b} K_{1}(\tau, x) d \tau, G_{0}=\sup _{x \in(a, b)} \int_{a}^{b} K_{2}(\tau, x) d \tau .
$$


Let us estimate the repeated integrals (18) in the expression for $\Phi(t)$, using (22), equality $K_{1}(\tau, x)=$ $=K(\tau, x)+K_{2}(\tau, x)$, inequality $\pm 2 c d \geq-\left(c^{2}+d^{2}\right)$ as well as symmetry and non-negativity of the kernels $K_{1}(\tau, x), K_{2}(\tau, x)$.

Using the conditions (5), we obtain

$$
\begin{gathered}
J_{i}(t) \geq-\frac{\rho V^{2}}{\pi} \int_{a}^{b} d x \int_{a}^{b}{w_{i}^{\prime}}^{2}(x, t) \times \\
\times\left|K_{1}(\tau, x)+g_{1}(x)+g_{1}(\tau)\right| d \tau, i=1,2, \\
J_{3}(t) \geq-\frac{\rho V^{2}}{\pi} \int_{a}^{b} d x \int_{a}^{b}\left(w_{1}^{\prime 2}(x, t)+{w_{2}^{\prime}}^{2}(x, t)\right) \times \\
\times\left|K_{2}(\tau, x)+g_{2}(x)+g_{2}(\tau)\right| d \tau,
\end{gathered}
$$

where $g_{1}(x), g_{2}(x)$ are the arbitrary functions integrable over a segment $[a, b]$.

Using the condition (9), we obtain

$$
\begin{aligned}
\sum_{i=1}^{3} I_{i}(t) & \geq \sum_{i=1}^{2} \int_{a}^{b} d x \int_{a}^{b} \dot{w}_{i}(x, t) \dot{w}_{i}(\tau, t) K_{2}(\tau, x) d \tau+ \\
+I_{3}(t) & \geq-2 \int_{a}^{b} d x \int_{a}^{b}\left(\dot{w}_{1}^{2}(x, t)+\dot{w}_{2}^{2}(x, t)\right) \times \quad(30) \\
& \times\left|K_{2}(\tau, x)+g_{2}(x)+g_{2}(\tau)\right| d \tau .
\end{aligned}
$$

Thus, according to (17), (18), (28) - (30), we obtain the inequality

$$
\begin{array}{r}
\Phi(t) \geq \sum_{i=1}^{2} \int_{a}^{b}\left\{E_{i} F_{i}\left(u_{i}^{\prime}+\frac{1}{2}{w_{i}^{\prime}}^{2}\right)^{2}+\right. \\
+\left(M_{i}-\frac{2 \rho K}{\pi}\right) \dot{w}_{i}^{2}+M_{i} \dot{u}_{i}^{2}+D_{i} w_{i}^{\prime \prime 2}+\beta_{0 i} w_{i}^{2}- \\
\left.-\left(\frac{\rho V^{2}(G+K)}{\pi}+N_{i}(t)\right){w_{i}^{\prime}}^{2}\right\} d x
\end{array}
$$

from which, using the first inequalities from (15), (16) and the second inequality from (20), we obtain

$$
\begin{array}{r}
\Phi(t) \geq \sum_{i=1}^{2} \int_{a}^{b}\left(D_{i} \lambda_{1 i}-\right. \\
\left.-\frac{\rho V^{2}(G+K)}{\pi}-N_{i}(t)\right){w_{i}^{\prime}}^{2} d x .
\end{array}
$$

Let the second condition (16) be fulfilled, then, using the third inequality (20), taking into account (21), (27), (31), we obtain the inequality

$$
\sum_{i=1}^{2}\left(D_{i} \lambda_{1 i}-\frac{\rho V^{2}(G+K)}{\pi}-N_{i}(t)\right) \frac{w_{i}^{2}(x, t)}{(b-a)} \leq
$$

$$
\begin{aligned}
& \leq \sum_{i=1}^{2} \int_{a}^{b}\left\{E_{i} F_{i}\left(u_{0 i}^{\prime}+\frac{1}{2} w_{0 i}^{\prime 2}\right)^{2}+M_{i} \dot{u}_{0 i}^{2}+\right. \\
& +\left(M_{i}+\frac{\rho\left(K_{0}+G_{0}\right)}{\pi}\right) \dot{w}_{0 i}^{2}+D_{i} w_{0 i}^{\prime \prime}{ }^{2}+ \\
& \left.+\left(\frac{2 \rho V^{2} G_{0}}{\pi}-N_{i}(0)\right) w_{0 i}^{\prime 2}+\beta_{0 i} w_{0 i}^{2}\right\} d x .
\end{aligned}
$$

Thus, if the conditions (15), (16) are satisfied, then $\Phi(t) \geq 0, \dot{\Phi}(t) \leq 0$. From (32) it follows that the solution $w_{i}(x, t)(i=1,2)$ of the system of equations (13) is stable with respect to the perturbations of the initial values $w_{i}(x, 0), \dot{u}_{i}(x, 0), \dot{w}_{i}(x, 0), u_{i}^{\prime}(x, 0), w_{i}^{\prime}(x, 0)$, $w_{i}^{\prime \prime}(x, 0)(i=1,2)$.

The theorem is proved.

Remark. In a similar way, we can prove that theorem 2.1 is also true, if the functions $u_{i}(x, t), w_{i}(x, t), i=$ 1,2 satisfy any combination of the following boundary conditions:

1) rigid fastening:

$$
w_{i}\left(x_{1}, t\right)=w_{i}^{\prime}\left(x_{1}, t\right)=u_{i}\left(x_{1}, t\right)=0 ;
$$

2) hinged fastening:

$$
w_{i}\left(x_{1}, t\right)=w_{i}^{\prime \prime}\left(x_{1}, t\right)=u_{i}\left(x_{1}, t\right)=0,
$$

where $x_{1}=a$ or $x_{1}=b$.

\subsection{An Example of a Mechanical System}

Let us give examples of calculating the stability region on the plane of two parameters $\left(V, N_{i}\right)$. We assume that the flow of liquid $(\rho=1000)$, and the elements are made of steel $\left(E_{i}=20,6 \cdot 10^{10}, \rho_{n i}=7850\right)$. Other parameters of the mechanical system: $a=0 ; b=1 ; y_{0}=1$; $\nu_{i}=0,25 ; \beta_{0 i}=4 ; \beta_{1 i}=0,4 ; \beta_{2 i}=0,4 ; h_{i}=0,01$ (all values are given in the SI system). Making calculations we obtain $\lambda_{1 i}=4 \pi^{2} ; M_{i}=78,5 ; D_{i} \approx 18311,1$.

According to conditions (16) it is necessary to find the coefficients $K, G$. For the calculation, the functions

$$
\begin{array}{r}
g_{1}(x)=-1,25 \sin \frac{\pi x}{2}+0,23 \cos \frac{\pi x}{2}-0,37, \\
g_{2}(x)=-\frac{\pi x}{2}-0,21 \sqrt{x(1-x)}+0,198
\end{array}
$$

were selected and the coefficients (14): $K \approx 0,243$; $G \approx 0,956$ were found using the Mathcad mathematical system. The first condition (16) is satisfied, and from the second condition we obtain

$$
N_{i}(t) \leq 722893,252-381,654 \cdot V^{2} .
$$

If the points $\left(V, N_{i}(t)\right)$ for any $t \geq 0$ do not go beyond the stability region (gray area in Figure 3 below the parabola), then the solutions $w_{i}(x, t), i=1,2$ of the system of equations (13) are stable with respect to the perturbations of the initial values $w_{i}(x, 0), \dot{u}_{i}(x, 0)$, $\dot{w}_{i}(x, 0), u_{i}^{\prime}(x, 0), w_{i}^{\prime}(x, 0), w_{i}^{\prime \prime}(x, 0), i=1,2$. 


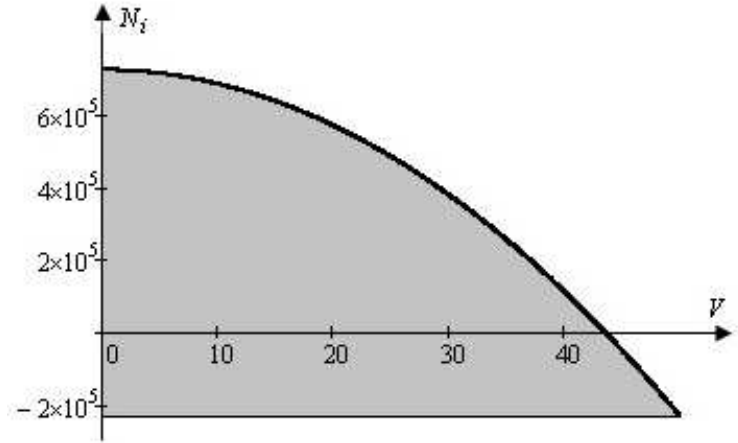

Figure 3. Stability region.

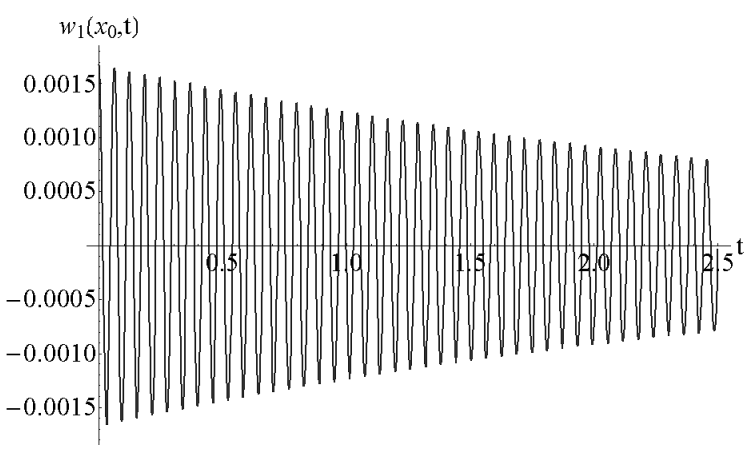

Figure 4. Character of the oscillations at $\mathrm{V}=40$.

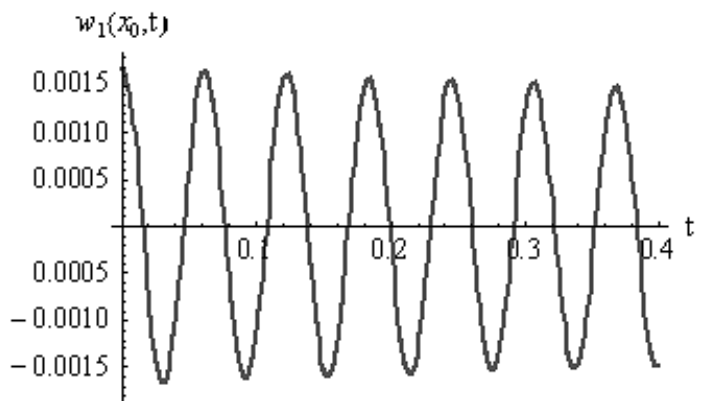

Figure 5. Character of the oscillations at $\mathrm{V}=40$.

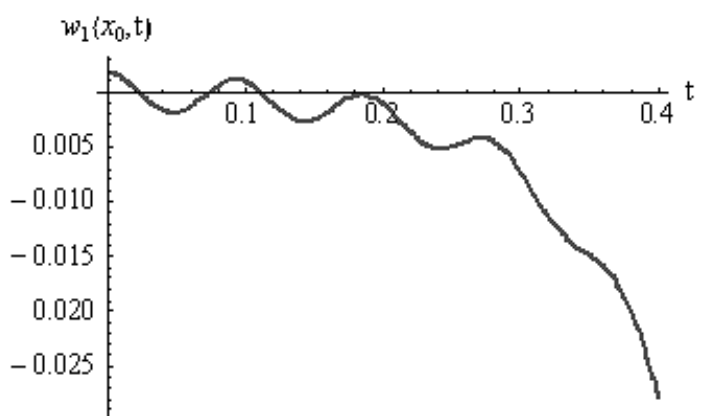

Figure 6. Character of the oscillations at $\mathrm{V}=51$.

\subsection{Numerical Experiment}

The solution of the system of equations (13) was found by the Galerkin-Petrov method, subjecting the desired functions $w_{i}(x, t), u_{i}(x, t)$ to the boundary conditions (5). At $N_{i}(t)=0$ in accordance with (16), the condition

$$
V<\sqrt{\frac{D_{i} \lambda_{1 i} \pi}{\rho(G+K)}} \approx 43,52
$$

must be fulfilled.

We take the initial conditions in the form

$$
\begin{array}{r}
u_{1}(x, 0)=\dot{u}_{1}(x, 0)=u_{2}(x, 0)=\dot{u}_{2}(x, 0)=0, \\
w_{1}(x, 0)=w_{2}(x, 0)=0,001 \psi(x), \\
\dot{w}_{1}(x, 0)=\dot{w}_{2}(x, 0)=-0,0005 \psi(x),
\end{array}
$$

where

$\psi(x)=\sin \gamma x-\sinh \gamma x-1,018(\cos \gamma x-\cosh \gamma x)$,

$\gamma=0,73$ (case of synchronous vibrations). The figure shows the character of the oscillations of the elastic elements at the point $x_{0}=1 / 2$ for the value $V=40$, that lies in the stability region, and the value $V=51$, that does not lies in the stability region. Obviously, at $V=40$ the stability is observed in the figures 4,5 and in the figure 6 - instability at $V=51$. The numerical experiments confirm the accuracy of the produced research.

\section{Model of Device with One Elastic Element}

We consider the model of device with one elastic element (Figure 7).

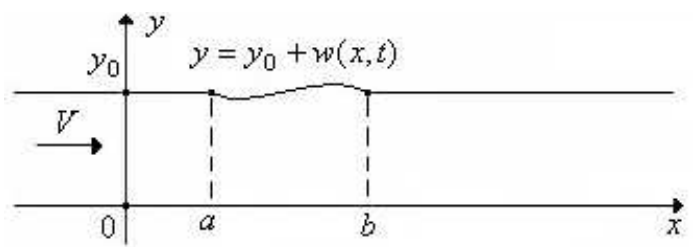

Figure 7. A channel whose wall contain deformable element

We obtain a special case the nonlinear boundary value problem (1) - (6) at $w_{1}(x, t)=0, w_{2}(x, t)=w(x, t)$, $u_{1}(x, t)=0, u_{2}(x, t)=u(x, t)$. Hence, from (13) we obtain a system of differential equations with two unknown functions $u(x, t), w(x, t)$ :

$$
\begin{gathered}
-E F\left(u^{\prime}(x, t)+\frac{1}{2}{w^{\prime}}^{2}(x, t)\right)^{\prime}+M \ddot{u}(x, t)=0, \\
-E F\left[w^{\prime}(x, t)\left(u^{\prime}(x, t)+\frac{1}{2} w^{\prime 2}(x, t)\right)\right]^{\prime}+ \\
+M \ddot{w}(x, t)+D_{i} w^{\prime \prime \prime \prime}(x, t)+N(t) w^{\prime \prime}(x, t)+ \\
+\beta_{0} w(x, t)+\beta_{1} \dot{w}(x, t)+\beta_{2} I \dot{w}^{\prime \prime \prime \prime}(x, t)= \\
=-\frac{\rho V}{\pi} \int_{a}^{b}\left(\dot{w}(\tau, t)+V w^{\prime}(\tau, t)\right) \frac{\partial K_{1}(\tau, x)}{\partial x} d \tau- \\
-\frac{\rho}{\pi} \int_{a}^{b}\left(\ddot{w}(\tau, t)+V \dot{w}^{\prime}(\tau, t)\right) K_{1}(\tau, x) d \tau, x \in(a, b) .
\end{gathered}
$$


Therefore, the theorem 2.1 takes the following form.

Theorem 3.1. Let the conditions

$$
\begin{array}{r}
\beta_{0} \geq 0, \quad \beta_{1}+\beta_{2} I \eta_{1} \geq 0, \quad \dot{N}(t) \geq 0, \\
M \geq \frac{\rho K}{\pi}, \quad N(t)<D \lambda_{1}-\frac{\rho V^{2} G}{\pi}
\end{array}
$$

be fulfilled. Then the solution $w(x, t)$ of the system of equations (34) is stable with respect to the perturbations of the initial values $w(x, 0), \dot{u}(x, 0), \dot{w}(x, 0), u^{\prime}(x, 0)$, $w^{\prime}(x, 0), w^{\prime \prime}(x, 0)$, if the functions $u(x, t), w(x, t)$ satisfy the boundary conditions

$$
\begin{array}{r}
u(a, t)=w(a, t)=w^{\prime}(a, t)=u(b, t)= \\
=w(b, t)=w^{\prime}(b, t)=0 .
\end{array}
$$

\section{Comparison with Finite Length Model}

In the paper [Velmisov and Ankilov, 2019] we considered a model of a device of finite length $x_{0}$ (Figure 8).

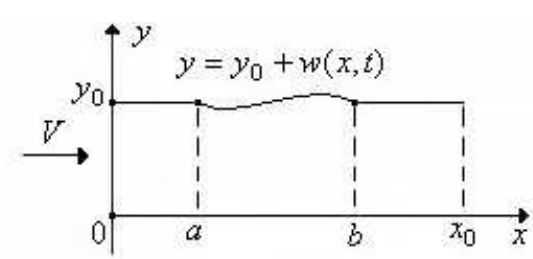

Figure 8. A channel whose wall contain deformable element

In the boundary sections of the channel the laws of variation of the longitudinal component of the fluid velocity are given:

$$
\phi_{x}(0, y, t)=0, \phi_{x}\left(x_{0}, y, t\right)=0, y \in\left[0, y_{0}\right], t \geq 0 .
$$

We introduce the following notation:

$$
\begin{aligned}
& G_{1}(\tau, x)=\ln \left|\frac{f(a)-f(b)}{f(\tau)-f(x)}\right|, \\
& G_{2}(\tau, x)=\ln \left|\frac{f(a)-f(b)}{f(\tau)+f(x)}\right|,
\end{aligned}
$$

where

$f(x)=c d\left(\frac{2 K(k) i\left(x_{0}-x\right)}{y_{0}}\right), c d x=-s n(x-K(k))$,

snx is the elliptic sine, $K(k)$ is the full elliptic integral of the first kind, the module $k$ is determined from the relation $K\left(\sqrt{1-k^{2}}\right) y_{0}=2 K(k) x_{0}$.

Then, using methods of the theory of functions of a complex variable, we obtained a system of differential equations with two unknown functions $u(x, t), w(x, t)$ :

$$
-E F\left(u^{\prime}(x, t)+\frac{1}{2} w^{\prime 2}(x, t)\right)^{\prime}+M \ddot{u}(x, t)=0,
$$

$$
\begin{gathered}
-E F\left[w^{\prime}(x, t)\left(u^{\prime}(x, t)+\frac{1}{2} w^{\prime 2}(x, t)\right)\right]^{\prime}+ \\
+M \ddot{w}(x, t)+D_{i} w^{\prime \prime \prime \prime}(x, t)+N(t) w^{\prime \prime}(x, t)+\quad(38 \\
+\beta_{0} w(x, t)+\beta_{1} \dot{w}(x, t)+\beta_{2} I \dot{w}^{\prime \prime \prime \prime}(x, t)= \\
=-\frac{\rho V}{\pi} \int_{a}^{b}\left(\dot{w}(\tau, t)+V w^{\prime}(\tau, t)\right) \frac{\partial G_{1}(\tau, x)}{\partial x} d \tau- \\
-\frac{\rho}{\pi} \int_{a}^{b}\left(\ddot{w}(\tau, t)+V \dot{w}^{\prime}(\tau, t)\right) G_{1}(\tau, x) d \tau, x \in(a, b) .
\end{gathered}
$$

Similarly to the above studies, based on the study of a functional of Lyapunov type, the following theorem is proved.

Theorem 4.1. Let the conditions (35), (36) be fulfilled, where

$$
\begin{aligned}
& G=\sup _{x \in[a, b]} \int_{a}^{b}\left|G_{1}(\tau, x)+g_{1}(x)+g_{1}(\tau)\right| d \tau, \\
& K=\sup _{x \in[a, b]} \int_{a}^{b}\left|G_{2}(\tau, x)+g_{2}(x)+g_{2}(\tau)\right| d \tau .
\end{aligned}
$$

Then the solution $w(x, t)$ of the system of equations (38) is stable with respect to the perturbations of the initial values $w(x, 0), \dot{u}(x, 0), \dot{w}(x, 0), u^{\prime}(x, 0), w^{\prime}(x, 0)$, $w^{\prime \prime}(x, 0)$, if the functions $u(x, t), w(x, t)$ satisfy the boundary conditions (37).

Compare the second condition (36) for the models in the figures 7 and 8 . We take the following parameters of the studied mechanical systems: $D=810 ; \rho=840$; $y_{0}=0.5 ; a=0.8 ; b=1.2$. Under conditions (37) the smallest eigenvalue $\lambda_{1}=25 \pi^{2}$. All values are given in the SI system.

For the model in the figure 7 , choosing a function $g_{1}(x)=-0.81-0.268 \cdot \sin (2.5 \pi(x-a))+0.493 \times$ $\times \cos (2.5 \pi(x-a))$, we find $G=0.371212$. The second condition (36) takes the form

$$
N(t)<199859.49-99.25 \cdot V^{2} .
$$

For the model in the figure 8 at $x_{0}=2$ we find the module $k=1.4 \cdot 10^{-5}$ from the equation $K\left(\sqrt{1-k^{2}}\right)=8 K(k)$. Choosing a function $g(x)=-0.42-0.268 \cdot \sin (2.5 \pi(x-a))+0.493 \times$ $\times \cos (2.5 \pi(x-a))$, we find $G=0.370818$. The second condition (36) takes the form

$$
N(t)<199859.49-99.15 \cdot V^{2} .
$$

The conditions are almost identical, which confirms the adequacy of the proposed mathematical model and the obtained results . 


\section{Conclusion}

On the basis of the proposed mathematical model of oscillations of elastic elements of a vibrating device in the form of a plate-strip with a single-sided flow around them by a subsonic flow of an ideal liquid, the investigation of the dynamics and stability of these elements was conducted. The obtained stability conditions impose restrictions on the mass and bending stiffness of elements, compressive (tensile) force elements, the velocity of an unperturbed flow and other parameters of the mechanical system. These conditions clearly contain the basic parameters of the mechanical system, and in this form they are most suitable for solving optimization problems, automatic control, and automated design.

\section{Acknowledgements}

The work was supported by the Russian foundation for basic research, grants 18-41-730015.

\section{References}

Abdelbaki, A. R., Paidoussis, M. P., and Misra, A. K. (2019). A nonlinear model for a hanging tubular cantilever simultaneously subjected to internal and confined external axial flows. Journal of Sound and Vibration, 449, pp. 349-367.

Ankilov, A. V. and Velmisov, P. A. (2013). Mathematical modeling in problems of dynamic stability of deformable elements of constructions at aerohydrodynamic influence. Ulyanovsk State Technical University, Ulyanovsk.

Ankilov, A. V. and Velmisov, P. A. (2016). Stability of solutions to an aerohydroelasticity problem. Journal of Mathematical Sciences (United States), 219 (1), pp. 14-26.

Blinkov, Y. A., Blinkova, A. Y., Evdokimova E. V. and Mogilevich, L. I. (2018). Mathematical modeling of nonlinear waves in an elastic cylindrical shell surrounded by an elastic medium and containing a viscous incompressible liquid. Acoustical Physics, 64 (3), pp. 274-279.

Kheiri, M. and Paidoussis, M. P. (2015). Dynamics and stability of a flexible pinned-free cylinder in axial flow. Journal of Fluids and Structures, 55, pp. 204-217.

Kollatz, L. (1968). Problems on eigenvalues. 503 p. Moscow. Science.

Kontzialis, K., Moditis, K. and Paidoussis, M. P. (2017). Transient simulations of the fluid-structure interaction response of a partially confined pipe under axial flows in opposite directions. Journal of Pressure Vessel Technology, Transactions of the ASME, 139 (3).

Moditis, K., Paidoussis, M. and Ratigan, J. (2016). Dynamics of a partially confined, discharging, cantilever pipe with reverse external flow. Journal of Fluids and Structures, 63, pp. 120-139.

Mogilevich, L. I., Popov, V. S., Popova, A. A., Christoforova, A. V. and Popova, E. V. (2017). Mathematical modeling of three-layer beam hydroelastic oscillations. Vibroengineering Procedia, 12, pp. 12-18.

Mogilevich, L. I., Popov, V. S. and Popova, A. A. (2018). Longitudinal and transverse oscillations of an elastically fixed wall of a wedge-shaped channel installed on a vibrating foundation. Journal of Machinery Manufacture and Reliability, 47 (3), pp. 227-234.

Moshkelgosha, E., Askari, E., Jeong, K.-H. and Shafiee, A. A. (2017). Fluid-structure coupling of concentric double FGM shells with different lengths. Journal of structural engineering and mechanics, 61 (2), pp. 231244.

Shmidt, G. (1978). Parametric Vibrations. 336 p. Moscow. Mir.

Velmisov, P. A. and Ankilov, A. V. (2017). Dynamic stability of plate interacting with viscous fluid. Cybernetics and physics, 6 (4), pp. 262-270.

Velmisov, P. A. and Ankilov, A. V. (2018). Stability of solutions of initial boundary-value problems of aerohydroelasticity. Journal of Mathematical Sciences (United States), 233 (6), pp. 958-974.

Velmisov, P. A. and Ankilov, A. V. (2018). Dynamic stability of deformable elements of designs at supersonic mode of flow. Journal of Samara State Tech. Univ., Ser. Phys. Math. Sci., 22 (1), pp. 96-115.

Velmisov, P. A., Gorshkov, G. M. and Ryabov, G. K. (1996). Hydrodynamic emitter. Patent 2062662, Russian Federation, MPK6 V 06 V 1/18, 1/20, Ulyanovsk state technical university, 5038746/28.

Velmisov, P. A. and Ankilov, A. V. (2019). Investigation of stability of elastic element of vibration device. In Proc. Int. Conf. "Stability, Control, Differential Games" (SCDG2019), Yekaterinburg, Russia, Sept. 16-20, pp. 91-95.

Zvyagin, A.V. and Gur'ev, K.P. (2017). A fluid-saturated porous medium under the action of a moving concentrated load. Moscow University Mechanics Bulletin, 72 (2), pp. 34-39. 